Case

Report

\title{
Recurrent Pneumomediastinum in Two Young Adults
}

Deok Heon Lee, MD, Gun-Jik Kim, MD, Youngok Lee, MD, and Sang Cjeol Lee, MD

\begin{abstract}
Spontaneous pneumomediastinum (SPM) is an uncommonly encountered entity. In addition, due to its rarity, little is known about recurrent SPM. We report on two exceptional cases of recurrent pneumomediastinum. One was a 16-year-old male whose first episode was accompanied by blunt chest trauma followed by a spontaneous second episode with a free interval of 17 months. The other case was a 17-year-old male whose presenting symptom was right and left pleuritic chest pain, respectively, without any predisposing or precipitating factors. We diagnosed these patients without performing invasive procedures and administered conservative management with success.
\end{abstract}

Keywords: pneumomediastinum, recurrence

\section{Introduction}

Spontaneous pneumomediastinum (SPM) is an infrequently encountered entity without obvious etiologies. Since the first description of SPM by Louis Hamman in 1939, many clinical series have reported its benign and self-limiting characteristics. ${ }^{1-4)}$ In addition, articles on recurrent SPM have occasionally been reported., ${ }^{3,5-7)}$ Incidence of recurrence is still vague, and it is estimated as being very low. Chest pain and dyspnea are the two most common symptoms. Subcutaneous emphysema can be found on physical examination. Patients may have a history of asthma, vomiting, or intensive sporting activity. However, predisposing factors and clinical manifestations are diverse and non-specific. Herein, we

Department of Thoracic and Cardiovascular Surgery, Kyungpook National University Hospital, School of Medicine, Kyungpook National University, Daegu, Korea

Received: May 23, 2013; Accepted: June 19, 2013

Corresponding author: Sang Cjeol Lee, MD. Department of Thoracic and Cardiovascular Surgery, Kyungpook National University Hospital, School of Medicine, Kyungpook National University, 130 Dongdeok-ro, Jung-gu, Daegu, Korea Email: sfelee@hanmail.net

(C)2014 The Editorial Committee of Annals of Thoracic and Cardiovascular Surgery. All rights reserved. report on two exceptional cases of recurrent pneumomediastinum.

\section{Case Report}

\section{Case 1}

A 16-year-old male dashed against another player while playing soccer. He felt anterior chest burning sensation after the collision. He visited a primary care hospital. Chest radiography and electrocardiography were unremarkable. That night, he was admitted to our emergency department for persistent anterior chest burning sensation and dysphagia. Vital signs and physical examination showed no specific findings. He had no special prior medical history, including asthma, allergy, illicit drug use, or smoking. Chest radiography showed air streaks in the upper mediastinum. Computed tomography (CT) scan confirmed pneumomediastinum without any other abnormalities (Fig. 1A). Considering the possibility of esophageal perforation, gastrograffin swallow was performed, with a negative result. Conservative treatment was administered, including one-day nil per os (NPO), antibiotics, and analgesics. On the second day, he was allowed to eat and was discharged with improvement of symptoms. After 17 months, he felt acute anterior chest pain while walking. He was admitted 

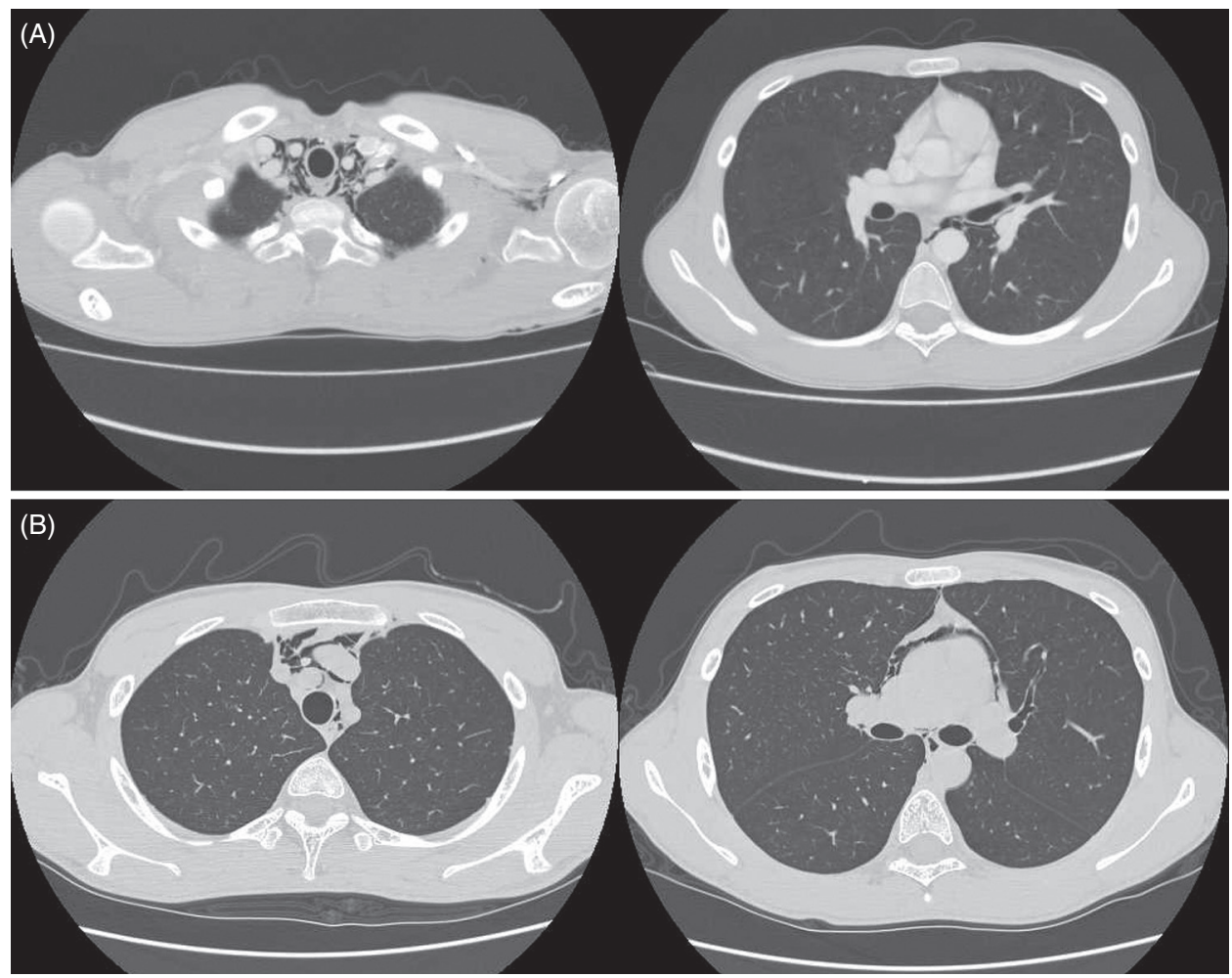

Fig. 1 (A) Computed tomography (CT) scan of the chest showing the presence of pneumomediastinum and interstitial air spreading along the bronchovascular bundles (the first episode of the first case). (B) Pneumomediastinum extending mainly through the anterior mediastinum (the second episode of the first case).

again with aggravated chest pain. There was no occurrence of triggering factors during this episode. Chest radiography and $\mathrm{CT}$ scan showed pneumomediastinum with no other pathologic findings (Fig. 1B). Subsequent esophagogram was normal. He received antibiotics and analgesics without NPO. He was discharged home after 1 day admission, and he was doing well without sequelae at the outpatient clinic 1 month later.

\section{Case 2}

A 17-year-old male was admitted to our emergency department for acute pleuritic left chest pain, which developed while just sitting at school. His medical history was unremarkable. He had not experienced any traumatic insult. In addition, he was neither a drug user nor a smoker. There were no precipitating factors, such as vomiting, coughing, activity associated with Valsalva's maneuver, or intensive exercise in this case. Subcutaneous emphysema was present in the neck. No evidence of intrathoracic disorders concerning viscus perforation or acute mediastinitis was observed on chest
CT scan (Fig. 2A). The only abnormal laboratory finding

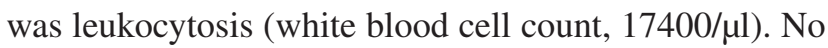
further investigations were performed. He was managed with analgesics alone without antibiotics and NPO. He was discharged home on the second hospital day after chest pain was relieved. After 7 months, he visited the emergency department again for abrupt right chest pain. Presenting symptoms and situation were similar to those of a previous event, except for laterality. Chest radiography and CT scan showed pneumomediastinum with otherwise normal findings (Fig. 2B). We applied the same management protocol i.e. no further diagnostic procedures, no NPO, and no antibiotics. His symptoms showed prompt resolution and he was discharged the next day. One week later, he appeared well in the outpatient clinic.

\section{Discussion and Conclusion}

SPM is an uncommon disorder defined as the presence of air in the mediastinum without obvious etiology. 

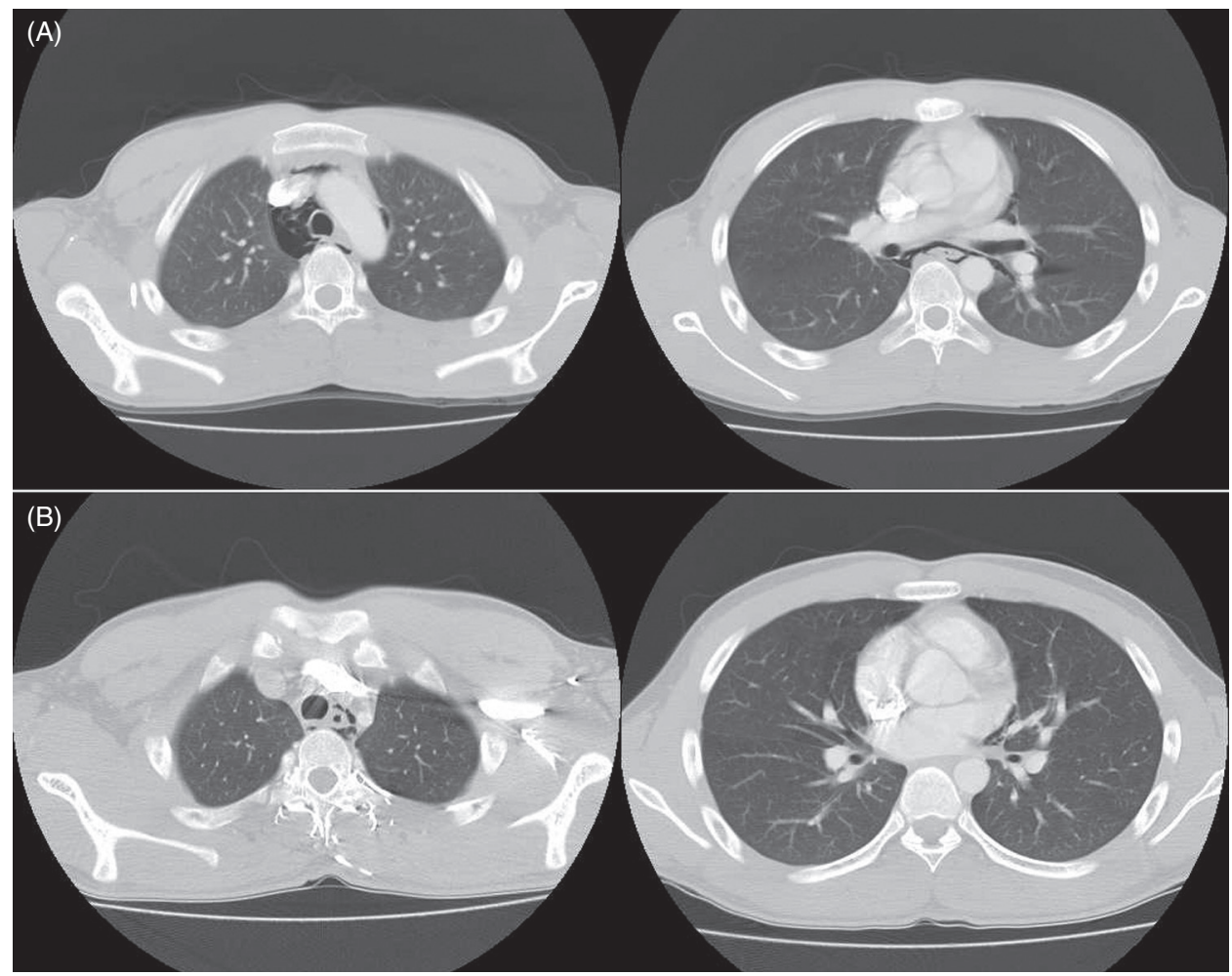

Fig. 2 (A) CT scan of the chest showing pneumomediastinum from the left fissure through the hilum to the upper mediastinum (the first episode of the second case). (B) Pneumomediastinum with lesser extent than the previous episode (the second episode of the second case).

The incidence of SPM has been reported as occurring in approximately 1 in 30000 emergency department referrals. ${ }^{2,3)}$ Recurrence of SPM has been reported sporadically. ${ }^{3,5-7)}$ The incidence of recurrence is estimated to be very rare. In a recent review of the literature, Natale, et al. found 13 cases of recurred SPM, including their own case. ${ }^{7)}$ However, it is still unknown the proportion of recurrent SPM to total number of SPM. In our experience, there were 35 cases of SPM in 376775 emergency department presentations between January 2003 and March 2013. Among them, two cases recurred at the 17th and 7th month after the first episode, respectively. Chest pain, which is typically retrosternal, and dyspnea are the two most common presenting symptoms. Anterior chest burning sensation in the first case resembled that of reflux esophagitis. Alternative right and left pleuritic chest pain mimicking that of spontaneous pneumothorax in the second case is also peculiar at the onset of SPM. SPM usually has a benign and favorable clinical course; however, secondary pneumomediastinum accompanied by esophageal perforation may have a fatal outcome. Therefore, determining whether or not pneu- momediastinum is spontaneous is mandatory. Due to concern about esophageal tearing in the first case, we performed esophagogram in both episodes. A spontaneous episode of pneumomediastinum following a traumatic episode has not yet been reported. It is also exceptional that patients with SPM do not have any triggering factor and precipitating symptom, which is so called primary or idiopathic, although bronchial hyperreactivity testing was not performed in our cases. Chest radiography and CT scan are considered gold standard methods for diagnosis of SPM.,23) Barium swallow is required if there is high suspicion of esophageal perforation. However, routine use of invasive procedures such as bronchoscopy or esophagoscopy is not recommended. Once the diagnosis of SPM has been established, conservative management is the main stem of treatment, which consists of rest, analgesics, and close observation. The use of antibiotics, oxygen therapy, and dietary restriction is controversial. In our cases, we made a diagnosis by taking a thorough medical history, physical examination, and chest radiography. We managed them conservatively with analgesics with or without antibiotics and NPO. 
Presenting symptoms resolved more quickly in the second episodes than in the first ones. After every episode, both patients could be discharged within 2 days, as soon as symptoms showed improvement. There was no occurrence of complications on the follow-up. In conclusion, recurrent SPM is extremely rare. SPM could be diagnosed by non-invasive methods and managed successfully by conservative treatment.

\section{Disclosure Statement}

All authors have no relevant financial relationships or conflicts of interest to disclose.

\section{References}

1) Hamman L. Spontaneous mediastinal emphysema.
Bull Johns Hopkins Hospital 1939; 64: 1-21.

2) Newcomb AE, Clarke CP. Spontaneous pneumomediastinum: a benign curiosity or a significant problem? Chest 2005; 128: 3298-302.

3) Macia I, Moya J, Ramos R, et al. Spontaneous pneumomediastinum: 41 cases. Eur J Cardiothorac Surg 2007; 31: 1110-4.

4) Takada K, Matsumoto S, Hiramatsu T, et al. Management of spontaneous pneumomediastinum based on clinical experience of 25 cases. Respir Med 2008; 102: 1329-34.

5) Channer KS, Roberts CJ, Jeyasingham K. Recurrent idiopathic pneumomediastinum. Postgrad Med J 1985; 61: 1073-5.

6) Fiorelli A, Messina G, Capaccio D, et al. Recurrent spontaneous pneumomediastinum: a rare but possible event! J Thorac Dis 2012; 4: 431-3.

7) Natale C, D'Journo XB, Duconseil P, et al. Recurrent spontaneous pneumomediastinum in an adult. Eur J Cardiothorac Surg 2012; 41: 1199-201. 